\title{
Abnormal refractoriness in patients with Parkinson's disease after brief withdrawal of levodopa treatment
}

\author{
John Harrison, Leslie Henderson, Christopher Kennard
}

\begin{abstract}
Two pairwise matched groups of patients with mild to moderate Parkinson's disease and a group of normal age matched controls were used to investigate the effects on simple and choice reaction time (RT) of brief withdrawal of levodopa therapy. Comparisons within and across these groups disclosed a selective effect of levodopa withdrawal. After about 12 hours of levodopa deprivation, patients exhibited an exaggeration of normal refractoriness, the well established tendency for RT to increase progressively as the delay between a response and the next imperative signal is reduced below $0.5 \mathrm{~s}$. Increased refractoriness was at least as great for simple as choice RT. Choice RT on trials involving repetition (as opposed to alternation) of the previous response, however, showed no tendency towards greater impairment by brevity of the recovery period or by withdrawal of medication, eliminating an effector based account. With longer recovery periods, RT was unaffected by medication; indeed, unmedicated patients were as fast as normal subjects under these conditions. Even at the briefest delays, fully medicated patients did not differ from normal controls. The paper concludes with a critical review of chronometric studies of medication effects in Parkinson's disease.
\end{abstract}

(F Neurol Neurosurg Psychiatry 1995;59:499-506)

Keywords: Parkinson's disease; reaction time; levodopa treatment

Academic Unit of Neuroscience, Charing Cross and Westminster Medical School,

Fulham Palace Road, London, W6 8RF, UK

$\mathrm{J}$ Harrison

L Henderson

C Kennard

Correspondence to:

Dr John Harrison, Academic

Unit of Neuroscience,

Charing Cross and

Westminster Medical

School, Fulham Palace

Road, London, W6 8RF,

UK.

Received 23 June 1994 and in final revised form

17 July 1995

Accepted 21 July 1995 nor the manipulation of dopaminergic replacement therapy within the patient population (table 1) have yielded entirely consistent RT effects.

A major focus of neuropsychological interest has been the search for task-specific impairments which might be informative about underlying deficits. To this end, many studies have compared classic simple RT (SRT), in which a single stimulus is repeatedly used to elicit an unvarying, predefined response with binary choice RT (2CRT), in which alternative responses have been assigned to each of a pair of stimuli, so that stimulus presentation not only serves as the temporal cue to respond but specifies which response is required.

Studies of the effects of dopaminergic medication on reaction times in Parkinson's disease have generally failed to satisfy the elementary canons of pharmacological research, either in their procedure for selecting subjects or in their experimental design. In the typical study, five to eight patients have been selected from the extremely heterogeneous parkinsonian population and investigated using a simple within subjects experimental design. Many studies have failed to control order of testing effects by the inclusion of counterbalancing or by additional control groups.

The relative insensitivity of RT measures to medication status in previous studies of Parkinson's disease led us to seek a way of increasing the demands made upon the subject's ability to sustain a high rate of response initiation. Various strands of evidence suggest that the execution of a response by patients with Parkinson's disease is much more likely to be impaired if it forms an element in a composite. For example, in a task merely requiring transfer of gaze to a novel target, the kinematics of the saccade appear normal in mild to moderate Parkinson's disease. Under instruction to respond with a series of saccades reciprocating between target and central fixation, the first saccade-notwithstanding its elicitation by an identical stimulus-takes much longer even for controls to initiate. Not only is this effect greater in patients with Parkinson's disease, the accuracy of their saccade is also very abnormal. ${ }^{16}$ Furthermore, Benecke et $a l^{17}$ have shown that when required to execute two movements in rapid sequence, patients with Parkinson's disease who have been deprived of their medication exhibit abnormally extended interresponse intervals, the second movement also 
Table 1 Studies examining effect of medication on RT and $M T$

\begin{tabular}{|c|c|c|c|c|c|c|c|c|c|}
\hline \multirow{3}{*}{ Study } & \multicolumn{3}{|l|}{ Study details } & \multicolumn{3}{|c|}{ Reaction time } & \multicolumn{3}{|c|}{ Movement time } \\
\hline & \multirow[b]{2}{*}{$\operatorname{Ref}$} & \multirow[b]{2}{*}{ Task } & \multirow{2}{*}{$\begin{array}{l}\text { No of } \\
\text { patients }\end{array}$} & & & & & & \\
\hline & & & & On & Off & Diff & On & Off & Diff \\
\hline Velasco and Velasco $\dagger$ & (4) & SRT & 16 & 388 & 454 & 66 NS & $105 \mathrm{~cm} / \mathrm{s}$ & $87 \mathrm{~cm} / \mathrm{s}$ & $-18^{\star \star}$ \\
\hline Rafal et al & (5) & SRT & 9 & 480 & 510 & 30 NS & - & 一 & - \\
\hline & (5) & CRT & 8 & 665 & 895 & $230^{\star}$ & - & - & \\
\hline Girotti et al & $\begin{array}{l}(6) \\
(6)\end{array}$ & $\begin{array}{l}\text { SRT } \\
7 \text { CRT }\end{array}$ & $\begin{array}{l}21 \\
21\end{array}$ & $\begin{array}{l}395 \\
452\end{array}$ & $\begin{array}{l}449 \\
494\end{array}$ & $\begin{array}{l}54 \text { NS } \\
42 \text { NS }\end{array}$ & $\begin{array}{l}457 \\
458\end{array}$ & $\begin{array}{l}487 \\
567\end{array}$ & $\begin{array}{c}30^{\star \star} \\
109^{\star}\end{array}$ \\
\hline Bloxham et al & (7) & SRT & 10 & 365 & 383 & 18 NS & - & - & - \\
\hline Viallet et al & (8) & SRT & $5 / 9$ & 210 & 264 & $54^{\star}$ & 247 & 323 & $76^{\star}$ \\
\hline Pullman et al & (9) & SRT & 5 & 355 & 375 & $20 \mathrm{NS}$ & 260 & 315 & 59 \\
\hline & (9) & $2 \mathrm{CRT}$ & 5 & 380 & 410 & $30^{\star}$ & 280 & 330 & 64 \\
\hline Starkstein et al & (10) & $\begin{array}{l}\text { SRT } \\
\text { SRT }\end{array}$ & 7 & 329 & 341 & ${ }_{\partial \star \star}^{12} \mathrm{NS}$ & 281 & 369 & $\begin{array}{l}88^{\star} \\
\partial \star \star \star\end{array}$ \\
\hline $\begin{array}{l}\text { Montgomery et al } \\
\text { Pullman et alt }\end{array}$ & $\begin{array}{l}(11) \\
(12)\end{array}$ & $\begin{array}{l}\text { SRT } \\
\text { SRT }\end{array}$ & $\begin{array}{l}8 \\
5\end{array}$ & $? 331$ & 373 & 42 NS & 280 & 339 & $50^{\star \star \star}$ \\
\hline & (12) & 2 CRT & 5 & 371 & 464 & 93 NS & 284 & 340 & $60^{\star \star \star}$ \\
\hline Jordan et al & (13) & SRT & $32 / 34$ & 355 & 360 & $5 \mathrm{NS}$ & - & - & - \\
\hline & (13) & $2 \mathrm{CRT}$ & $32 / 34$ & 450 & 470 & $20 \mathrm{NS}$ & - & - & - \\
\hline Jahanshahi et al & (14) & SRT & 8 & 488 & 504 & 16 NS & 332 & 389 & $\begin{array}{l}57 \mathrm{NS} \\
25 \mathrm{NS}\end{array}$ \\
\hline Brown et al & $\begin{array}{l}(14) \\
(15)\end{array}$ & 2 CRT & $\begin{array}{l}8 \\
7\end{array}$ & 440 & $\begin{array}{l}020 \\
495\end{array}$ & $\begin{array}{l}-13 \mathrm{NS} \\
55^{\star}\end{array}$ & 4 & 409 & - \\
\hline & (15) & $2 \mathrm{CRT}$ & 7 & 440 & 490 & $50 \mathrm{NS}$ & - & - & - \\
\hline & (15) & $2 \mathrm{CRT}$ & 7 & 555 & 560 & 5 NS & - & - & - \\
\hline Harrison et al & (this study) & SRT & $8 / 8$ & 341 & 358 & $17^{\star}$ & - & - & - \\
\hline Harrison et al & (this study) & 2 CRT & $8 / 8$ & 379 & 411 & $32^{\star}$ & - & - & - \\
\hline
\end{tabular}

${ }^{\star} \mathrm{p}<0.05 ;{ }^{\star \star} \mathrm{p}<0.01 ;{ }^{\star \star \star} \mathrm{p}<0.001$.

Figures in italics have been estimated from graphs. The figure preceding CRT in Task column denotes the number of choice options presented in that study.

All responses are button press or release with finger with the exception of: †finger press or foot press; ‡wrist rotation; §move lever.

being of reduced velocity. Restoration of drug treatment greatly reduced both of these abnormalities without quite eliminating them.

Previous chronometric studies of performance in patients with Parkinson's disease have used comparatively long intertrial intervals. By contrast, we employed a microcomputer controlled, serial RT procedure in which the delay between a response and the onset of the imperative signal for the next trial was manipulated without intervention of a separate warning signal. This allowed us to measure SRT and 2CRT with response-stimulus intervals as brief as $\mathbf{5 0 ~ m s}$.

\section{Methods}

DESIGN

We employed a design in which the same reaction time measures were applied to three groups-two Parkinson's disease groups and a normal control group (fig 1). Each group undertook two experimental sessions on the same morning. Carefully screened patients with Parkinson's disease with mild to moderate motor impairments were matched pairwise on various clinical measures. One member of the pair was assigned at random to an experimental (PD:E) group and the other to a control (PD:C) group. Overnight withdrawal of

Figure 1 RT paradigms. (A) Classic simple RT, (B) choice RT with high compatibility. Visual stimuli versions of tasks $(A)$ and $(B)$ were used in this experiment.

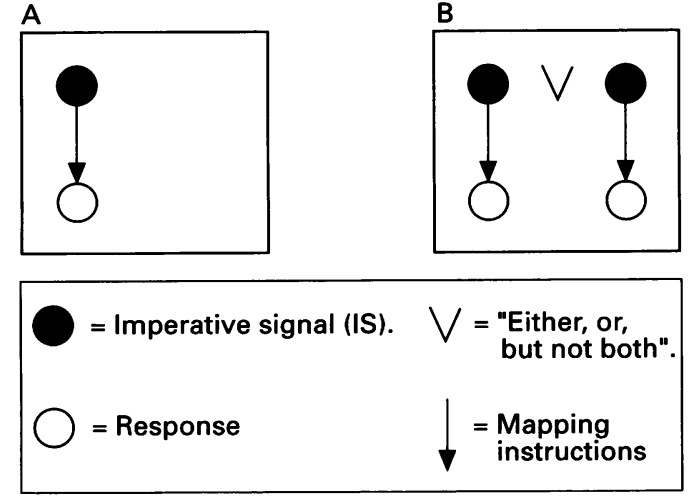

dopaminergic medication was confined to the PD:E group on the first session of testing. One hour later, after taking medication, they were admitted to the second session. This manipulation allowed us to assess the effects of medication by means of within group, as well as between group, comparisons, controlled for order of testing effects. The addition of a group of normal age matched controls allowed us to compare the differences attributable to medication with any differences between fully medicated patients with Parkinson's disease and normal subjects of the same age. This design also permits the assessment of any differential change in performance across sessions.

\section{SUBJECTS}

Eight closely matched pairs of subjects with a clear diagnosis of idiopathic Parkinson's disease were recruited from the outpatient populations of the Royal London and Charing Cross Hospitals. All had satisfied exclusion criteria for age, dementia, disease duration, symptom severity, and drug treatment (fig 2). Preliminary interview and testing had enabled subjects to be matched pairwise on age, duration of disease, and abbreviated Webster score. One member of each pair, determined randomly, was allocated to the PD:E group, the other to the PD:C group. A third group comprised eight normal age matched controls who had no history of neurological or psychiatric disease and were not taking medication known to affect the CNS.

\section{APPARATUS}

The two response buttons, $15 \mathrm{~cm}$ apart, were positioned on a table in front of the subject such that the forearms could be laid flat with the index fingers positioned one on either button. Beyond and adjacent to each button, a light delivered its imperative signal. For each trial the appropriate light remained on until a response was detected. A microcomputer con- 
Figure 2 Screening procedure and matching of subjects.
Screening - subject excluded if:

(1) Older than 75 years

(2) Demented (less than 27/30 on MMS)

(3) History of any neurological disorder (other than PD for patients)

(4) Receiving medication known to affect CNS (other than levodopa, dopamine agonists, and MAOB inhibitors in the case of PD)

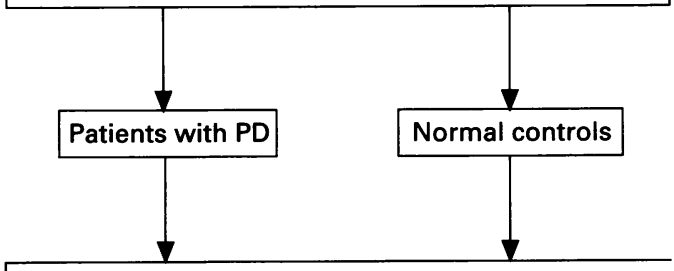

Subjects matched on the basis of;

(1) Age

(2) Sex

(3) Webster score (PD only)

(4) Disease duration (PD only)

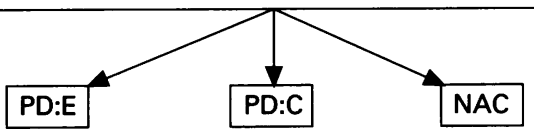

trolled the sequence and timing of stimuli and registered the responses, tabulating errors, and latencies.

\section{PROCEDURE}

Before each task, subjects were provided with a block of 21 practice trials. The two experimental sessions were separated by one hour, a period intended to be sufficiently long to allow the levodopa to take effect in the PD:E group. In each session, subjects performed an SRT and a two choice CRT (2CRT) task. Each task was given as a single block of 196 trials, composed of 28 replications of each of the seven levels $(50,100,200,400,800$, $1600,3200 \mathrm{~ms}$ ) of response-stimulus interval, in pseudorandom sequence. The correct (left or right) keypress response was unpredictable in CRT blocks. To balance the hand factor, subjects were instructed to change hands midway through the SRT task. The order of testing was counterbalanced across subjects within groups.

\section{Results}

For all three groups the error rate was negligible, never exceeding $1 \%$ for any block of trials. A preliminary analysis was directed towards the question of whether the attempt to match the two Parkinson's disease groups was reflected in equivalent performance in the second session, when both were fully medicated. Analysis of variance (ANOVA) with group, RT task, and response-stimulus interval as factors confirmed that neither the main effect of group $(F(1,14)<1 \cdot 0)$ nor any interaction involving the group factor approached significance $(P>0.20, \quad$ in all cases $)$. Significant main effects of task ( $F$ $(1,21)=26 \cdot 7, \quad P<0.0001)$ and responsestimulus interval $(F \quad(6,126)=25 \cdot 8$, $P<0.0001)$ were found, although they showed no tendency towards interaction ( $F$ $(6,126)<1 \cdot 0)$. Table 2 summarises the SRT
Table 2 Group by $R T$ task means for all three groups in session 2 (in which PD:Es were medicated

\begin{tabular}{lll}
\hline & $S R T$ & $C R T$ \\
\hline PD:E & $341(95)$ & $379(79)$ \\
PD:C & $339(64)$ & $369(64)$ \\
NAC & $334(79)$ & $376(52)$ \\
\hline
\end{tabular}

Values in parentheses are SD

and CRT latencies for both Parkinson's disease groups and also for controls. The differences in overall mean RT between the groups spanned a mere $6 \mathrm{~ms}$, with the normal age matched control group intermediate between the two Parkinson's disease groups.

We determined the absence of reliable differences between the performance of fully medicated patients with mild to moderate Parkinson's disease and normal healthy controls by subjecting the latencies of the PD:C and normal age matched control groups on both sessions to ANOVA. Again, neither the main effect of group $(F<1)$ nor any interaction involving the group factor were significant. In particular, the slight reduction in latency across the two sessions $(10 \mathrm{~ms}$ for PD:Cs and $12 \mathrm{~ms}$ for normal age matched controls) showed no differential tendency towards improvement $(F<1)$.

With this as a basis, we were able to proceed to our principal test, comparing the performance of the two Parkinson's disease groups in session 1 when the experimental group were off medication and in session 2 when their medication had been restored. The ANOVA disclosed a significant interaction of group, session, and response-stimulus intervals $(F(6,84)=2 \cdot 22, P<0.05)$. Figure 3 illustrates this. The source of this interaction seems to be the withdrawal of medication since it consists of a difference in performance which sets the PD:E group apart from the other groups only in session 1, when they were unmedicated. The effect consists of a latency prolongation, confined to the briefer response-stimulus intervals. Withdrawal of medication seems to exaggerate the normal tendency for latencies to increase, as if the subject requires some time to "recover" from the previous response. This "refractoriness" seemed to be abnormally increased by dopamine depletion.

We examined this differential effect of response-stimulus interval further, confining attention to a comparison of the performance of the two Parkinson's disease groups in session 1, when their state of medication differed. In view of previous reports of differential effects of medication on SRT and CRT we decided to include RT task as a factor in our analysis, despite its failure to interact with any of the other factors in the preceding ANOVA. As it seemed likely that a full breakdown of the data into individual responsestimulus intervals bins would leave too few observations per cell for statistical comparisons to be robust, we dichotomised the seven response-stimulus intervals into "long" $(0 \cdot 8-3 \cdot 2 \mathrm{~s})$ and "short" (50-200 ms), discarding the median value. Whereas the first 
Figure $3 P D$ group (PD:E v PD:C) $\times$ session $\times$ response-stimulus interval interaction $(F$ $(6,84)=2 \cdot 22, P<0 \cdot 05)$
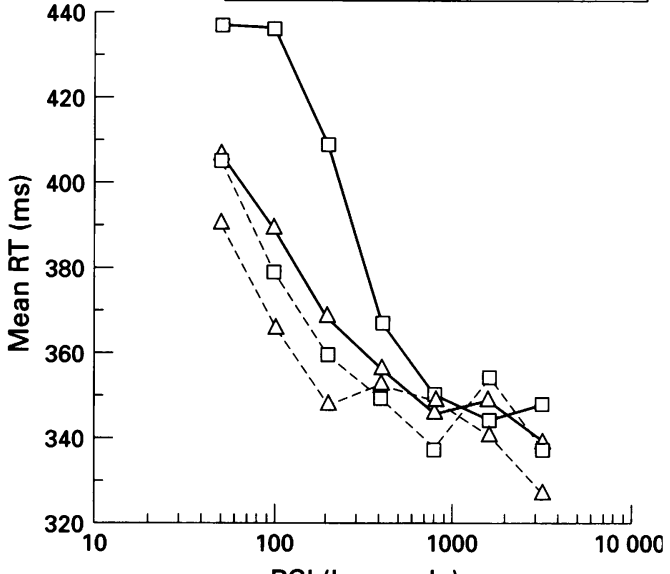

RSI (Log scale)

order group $\times$ response-stimulus interval interaction failed to attain significance, the group $\times$ RT task $\times$ response-stimulus interval interaction did prove significant $(F(1,14)=$ $4 \cdot 8, P<0.05)$. The form of this interaction can be inspected in fig 4, from which it seems that SRT at brief response-stimulus interval values best discriminated medication status. The negative outcome of further post hoc tests, however, prevented us from locating the medication effect more decisively.

Finally, we performed a sequential analysis of the session 1 CRT data, classifying every trial (except the first) in a block as either a repetition, involving the same stimulusresponse linkage as the preceding trial, or an alternation, to determine whether using the same effector twice within a brief interval was particularly difficult, especially for poorly medicated patients with Parkinson's disease. Repetition/alternation was then entered as a factor into an ANOVA, together with group and response-stimulus interval. This showed a surprising but highly significant $21 \mathrm{~ms}$ latency advantage for alternations $(F(1,20)=15 \cdot 4$, $P<0.001)$. An unambiguous lack of significant interactions involving repetition, however, suggested that any effects of medication, disease, or refractoriness were not qualified by repetition.

Figure 4 Difference between PD:E and PD:C groups in mean SRT and CRT latency for long and short response-stimulus intervals in session 1.

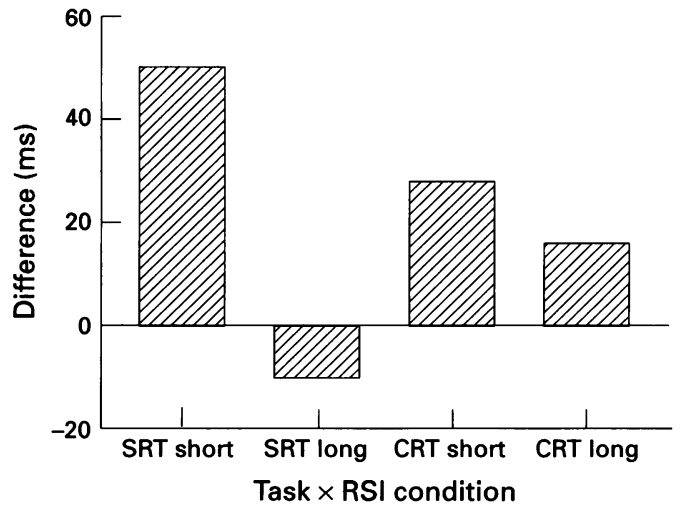

\section{Discussion}

In a serial RT study characterised by a relatively large number of observations per subject and the employment of briefer response-stimulus intervals than previously investigated in patients with Parkinson's disease, we were able to detect deleterious consequences even of brief levodopa withdrawal in mildly impaired patients. These took the form of an exaggeration of normal psychological refractoriness-a prolongation of RT that emerges as the recovery period between the response just executed and the signal summoning the next response is reduced below $0.5 \mathrm{~s}$. By embedding these features in a design more rigorous than any previously reported, involving two sessions of testing conducted on two pairwise matched Parkinson's disease groups and a normal age matched control group, we were able to exclude uncontrolled variables that pervade the literature on medication effects in Parkinson's disease. This allowed us to identify conditions which permit the detection of RT abnormalities consequent on levodopa withdrawal in patients whose RT performance, when fully medicated, is apparently normal.

\section{REFRACTORINESS AND PREPARATION FOR ACTION}

Despite 40 years of psychological enquiry, the phenomenon of "psychological refractoriness" is not well understood. The traditional account was focused on the possibility of competition between inputs for limited perceptual processing resources. The processes serving perceptual discrimination were thought to be the locus of this limitation. Since simple RT was assumed merely to require stimulus detection rather than full discrimination and identification, this meant that performance on the SRT task was not expected to involve competition for the resources of the limited capacity perceptual identification system and therefore would not be susceptible to refractoriness. As applied to CRT, the original focus of the limited capacity central processor hypothesis was on the latency prolongation in overlap paradigms where stimulus 2 arrives before the execution of response 1 . When it became evident that refractoriness, albeit diminished in magnitude, could be found even when stimulus 2 followed response 1 , proponents of the traditional interpretation were obliged to assume that the perceptual demands of an RT trial could extend beyond the execution of the response, due, perhaps, to the time taken to clear the central processor of the feedback generated by responding. ${ }^{18}$ However, even when the perceptual competition hypothesis is stretched in this way, it fails to accommodate the fact that refractoriness can extend to almost $0.5 \mathrm{~s}$ after the execution of a previous response (for example, Wilkinson ${ }^{19}$ and this study). Moreover, it seems clearly evident that some fixed limit on the rate of responding (response 1 -response 2 interval) is not operative, with perfect trading between the stimulus 2 -response 2 interval (RT in the current 
trial) and the preceding response 1 -stimulus 2 interval. Some critics have regarded this consideration as fatal for the perceptual bottleneck account.

An alternative interpretation of the competition between overlapping processes which gives rise to refractoriness involves a shift of emphasis from the lingering traces of perceptuocognitive activity on trial $\mathrm{T} 1$ to the benefits to be gained when the subject is allowed to devote sufficient time and undivided attention to preparation in advance for T2. A version of this account which locates this preparation towards the response end of the processing cascade and consequently declines to regard refractoriness as exclusively attached to CRT, has been advanced by Rabbitt. ${ }^{20}$ His thesis is that refractoriness is caused by interference of response 1 with the response preparation for T2, on which optimal RT depends. Such an account is vulnerable to any demonstration of a dissociation between the function describing improvement in RT with increasing foreperiod (warning interval) and the function showing recovery of $\mathrm{RT}$ with increases in response-stimulus interval. Wilkinson ${ }^{19}$ has proposed sleep deprivation as such a dissociating factor, as it changes the form of the preparation function, but not the refractoriness function. Whereas neither the manipulation of informative precues that reduce some of the subject's uncertainties about the required response nor manipulations of the timing of a warning signal which serves a general alerting function have allowed the identification of a defect of preparation in Parkinson's disease, studies of the ability to capitalise on the certitudes that are peculiar to the SRT task have suggested that this aspect of preparation is defective in a subset of patients with Parkinson's disease. ${ }^{21}$

In the present data, the undiminished magnitude of the refractoriness found in simple, as opposed to choice, RT is inconsistent with an interpretation of refractoriness in terms of central processes of stimulus discrimination or response selection. On the other hand, the failure to find greater refractoriness in CRT on trials in which the response is the same as that executed on the previous trial, seems to rule out an account in terms of limitations on response rate located at the effector level. The overall tendency in our CRT data towards briefer latencies on alternations than on repetitions seems most likely to be the result of a strategic bias induced by the time pressure of the refractoriness paradigm as in standard paradigms repetition has been widely found to be facilitatory.

What emerges from these considerations is the complexity of the issues surrounding preparation for rapid action. To the extent that refractoriness is interpretable as interference with preparation, the present experiment suggests that the process involved is vulnerable to levodopa withdrawal in Parkinson's disease and that this sensitivity might be greater in the SRT task. A quite separate strand of evidence suggests that some members of the parkinsonian population have lost the ability to engage in whatever preparations normally give SRT its latency advantage over high compatibility 2CRT. ${ }^{21}$ Recently, moreover, the finding that transcranial magnetic stimulation of motor cortex can reduce the abnormal prolongation of RT sometimes found in patients with Parkinson's disease. ${ }^{22} 23$ has provided quite a different sort of basis for inferring defective preparation. Because yet other paradigms have been used to argue for the intactness of (some) preparatory processes in Parkinson's disease, it remains to be seen whether these differences in findings across the range of paradigms are consistently found and if so how successfully they can be interpreted in terms of functionally distinct aspects of preparation for rapid action. Certainly, the studies have differed in regard to the impairments the subjects have brought to the experiment. Whereas the excessive refractoriness revealed by medication withdrawal in this study was obtained for patients with Parkinson's disease who showed no signs of RT abnormality when properly medicated, to demonstrate the absence of SRT advantage clearly entails abnormally prolonged SRT. Finally, in as much as the transcranial magnetic stimulation studies focus on the return of SRT and CRT to normality, an initial deficit in one or the other is presupposed.

\section{RT AS A MARKER FOR AKINESIA}

It is evident from the present results that RT conditions which are demonstrably capable of showing significant effects of medication withdrawal may nevertheless fail to discriminate the performance of fully medicated patients with Parkinson's disease from controls. Pooling the session 2 data for both Parkinson's disease groups, their overall mean RT ( $n=16$ ) exceeded that of the control group $(n=8)$ by a mere $4 \mathrm{~ms}$. Others have reported RTs in mild cases that are slightly but not significantly faster than controls. ${ }^{24}$ It seems incontestable, therefore, that, within the sort of mild-to-moderately impaired population of patients with Parkinson's disease that we have preferred to study, individuals can readily be found whose mean keypress $\mathrm{RT}$, based on an adequate number of observations, lies consistently well within normal bounds. We say "consistently" to emphasise that the individuals who are found to satisfy these criteria do not comprise an ever changing set that arise as the ephemeral products of random error variance; each subject in the present study generated about 800 RT observations. Nor can this apparent normality be dismissed as a consequence of insensitive methods of testing, as it was these very methods that enabled us to detect a significant effect of medication withdrawal in the same patients. It is impossible to reconcile these findings with the conclusion reached by Jahanshahi $e \mathrm{al}^{3}$ in reviewing the literature on chronometric indicators of abnormality in Parkinson's disease, in which they assert that "RT in Parkinson's disease is always slower than in age matched controls." ${ }^{24}$ It is worth noting that three other studies have recently 
been published in which patients with Parkinson's disease have been shown to exhibit reaction time latencies no different from normal control subjects. ${ }^{21} 2425 \mathrm{We}$ return to the difficulty of formulating meaningful generalisations about the entire Parkinson's disease population, at the end of this discussion.

LEVODOPA WITHDRAWAL AND REACTION TIME The literature on medication effects in manual RT comprises 13 papers, including this one (table 1). All but one ${ }^{15}$ assessed SRT; six measured CRT, ${ }^{599^{12-14}}$ and seven provided a movement time (MT) measure. ${ }^{46812-14}$ Curiously, almost all the CRT studies confined themselves to binary choices and none systematically varied number of stimulusresponse alternatives. Table 1 also shows for each separate experiment the "medication effect" (difference between performance on and off medication) for SRT, CRT, and MT. Altogether 11 estimates of SRT and MT are available and 10 of CRT. The proportion of these attaining statistical significance was $25 \%$ (SRT), $40 \%$ (CRT) and $73 \%$ (MT). Strangely, there is no correlation between the magnitude of the effects and their significance nor between the numbers of patients studied and the significance of the effects. Ten of the 11 MT effects and 21 out of 22 RT effects were, however, in the expected direction. (Under these circumstances it seems unwise, as Jahanshahi et $a l^{14}$ and Pullman et $a l^{9}$ have done, to draw strong conclusions from negative findings.)

Considering first the RT values, we shall discuss reasons for discarding the extreme, outlying CRT value of $230 \mathrm{~ms}$ reported by Rafal et al. ${ }^{5}$ Two other values, one at either extreme, seem possibly anomalous - the 91 ms value reported for directional CRT by Pullman et $a l^{9}$ and the negative value reported for 4CRT by Jahanshahi et al. ${ }^{14}$ The interquartile range of the remainder is $20-50$ ms and that of the MT values is $50-76 \mathrm{~ms}$. Whereas comparisons across studies that differ as much as these in their design and in the nature of the tasks are clearly hazardous, it seems worth remarking that nothing in these data supports a general distinction between the sensitivity of SRT and CRT to dopaminergic manipulations.

Two studies ${ }^{9-12}$ stand apart, both because of the nature of the response required, a wrist rotation task, and the manner of manipulating state of medication (three levels of levodopa perfusion). Most of the remainder used manual keying responses combined with overnight withdrawal of all dopaminergic medication followed by morning testing, restoration of medication, and retesting. Obvious problems with such a simple, within group uncounterbalanced design are (a) the confounding of true effects of medication with effects due to retesting itself, and $(b)$ the lack of a normative baseline allowing the separate assessment of the extent to which optimum medication fully restores normal performance. In a variant of this design, Jahanshahi et $a l^{14}$ delayed retesting for several months, thus adding a fresh problem-namely, $(c)$ : the possible confusion of medication effects with an overall change in the patient's state.

In general, designs employing a between group comparison avoid the problems of retesting at the cost of introducing possible confusion of drug effects with inherent differences between the groups. Standard methods of minimising this problem involve prior matching of groups or, when the number of subjects is sufficiently large, random allocation to groups. Conversely, by electing to compare a de novo group of patients with Parkinson's disease with a stable medicated group, Jordan et al ${ }^{13}$ built into their study the likelihood of confounding, to the extent that characteristics other than treatment set apart such populations. The study by Viallet et $a l^{8}$ has a more severe form of the problem afflicting the design of Jordan et al. ${ }^{13}$ Their control group comprised five unmedicated patients with Parkinson's disease, four of whom had a disease duration of less than two years and the remaining one a duration of 28 years. Neither is it made clear why these patients were not receiving medication, nor was an attempt made to match the two Parkinson's disease groups.

Turning to outcome, only a minority of the studies yielded significant medication effects. Of these, the CRT effect reported by Rafal et $\mathrm{al}^{5}$ seems anomalously large. They required patients with Parkinson's disease to "move a heavy lever either to the left or right in response to a peripheral cue". The very large facilitatory effect of medication they obtained contrasts not only with findings in the rest of the literature but also with the non-significant effect that they reported for keypress SRT. On closer examination, it transpires that in the heavy lever task, the purported CRT measure of Rafal et $a l^{5}$ combines RT and MT. It seems distinctly possible, therefore, that much or all of their medication effect is attributable to time taken to execute the movement, itself. Incidentally, the provision of an $80 \%$ reliable response precue in this study also reduces the subject's response uncertainty before the imperative signal to a level that threatens to obscure the distinction between SRT and CRT.

Brown et al ${ }^{15}$ found a significant impact of medication upon performance in a CRT task but this only obtained when using a direct spatial mapping of stimuli on to responses. In two variants, in which the responses were specified by symbolic stimuli, no effect of medication was observed. The study carried out by Montgomery and Nuessen ${ }^{11}$ required an aimed movement to a target of varying size. Subjects were presented with an auditory "go" signal and the time at which the subject initiated their movement was recorded as the reaction time. Such a combination of an SRT procedure with the need for an accurate movement excursion seems likely to encourage preprogramming, supplying a possible reason for their robust effect of medication. 
OBJECTIVES?

It is not immediately evident what larger theoretical question many of these studies consider. The casual reader might be forgiven for supposing that their aim is merely to enquire into the utility of RT as an overall marker or test of "parkinsonism"; levodopa withdrawal is used simply as a manipulation of the global severity of impairment and RT regarded as an undifferentiated neuropsychological test of "akinesia". As we wish to suggest, to the contrary, that such investigations are interesting to the extent that they deviate from that aim, we examine each component of that putative aim, reviewing, in turn, the questions of statistical power and significance in evaluations of the effects of levodopa withdrawal, the potential informativeness of task manipulations and the various chronometric measures they enable, and the principles guiding the selection of patients for study.

\section{POWER AND SIGNIFICANCE IN LEVODOPA}

MANIPULATIONS

If attaining statistical significance is accorded overall primacy then the studies reviewed would have to be reckoned as incompetent instruments. There is a paucity of trials used to determine RT, few patients have been studied, and there is a lack of any principled basis for patient selection. This results in unreliable measures with insufficient statistical power to sustain robust conclusions, as well as a lack of clarity about the population to which these results might be expected to generalise. Overnight withdrawal of levodopa is, moreover, a relatively ineffective manipulation on the mild to moderately impaired patients used in many of these studies. The availability of more efficacious means of capturing a wide range of parkinsonian impairment, whether by comparison across patients at markedly different stages of the disease or by longer withdrawal of therapy, is scarcely in doubt. The ultimate limits are more likely to be set by ethical than by pharmacological considerations. Furthermore, were the impact of medication withdrawal to be the primary concern we suspect that this purpose would be better served by manipulation of a direct acting agonist such as apomorphine, which is not subject to the vagaries of absorption by the gut, transport across the blood-brain barrier, and metabolic transformation into a substance with affinity for dopamine receptors. These properties endow apomorphine with the ability to switch some patients with severely progressed disease from a state where they are profoundly "off" to fully "on", and its brief half life allows an almost equally sudden reversion to immobility. As a separate issue, there are also strong indications that movement time captures the parkinsonian impairment more reliably than unadorned RT measures (table 1).

These considerations bring out a certain triviality in the question of whether statistically significant effects can be obtained. Moreover, if such researches are to amount to more than a licensing of valid discriminatory measures of global parkinsonism that might be used, let us say, in a drug trial, then it must be appreciated that comparison of normal age matched controls with severely impaired patients with Parkinson's disease is not interchangeable with levodopa withdrawal as a manipulation, any more than RT and MT are interchangeable measures of "slowness".

The alternatives to levodopa sensitivity merit deeper thought. At least three other possibilities have been contemplated. Prolonged RT in Parkinson's disease might be a strategic adaptation to the motor damage in Parkinson's disease, ${ }^{26}$ it might be a nonspecific product of certain types of brain damage, ${ }^{14}$ or it might result from depletion of a neurotransmitter other than dopamine. For instance, a postmortem study ${ }^{27}$ has shown that brain noradrenaline, known to be related to attention and vigilance, ${ }^{28}$ is reduced in Parkinson's disease. One study of RT in Parkinson's disease ${ }^{29}$ has looked at the relationship between reaction time and CSF levels of the noradrenaline metabolite, 3-methoxy-4-hydroxyphenyletheneglycol (MHPG), yielding significant correlations with SRT, CRT and various measures derived from the continuous performance test. Unfortunately, the only studies in table 1 to consider non-dopaminergic sources of RT abnormalities in Parkinson's disease ${ }^{91214}$ seem to have been driven to this in extremis. Pullman et al, ${ }^{9}$ finding that when plasma levodopa was raised to a clinically defined criterion of "fully on" CRT was reduced by $91 \mathrm{~ms}$ and SRT by only $40 \mathrm{~ms}$, concluded post hoc that optimal SRT performance relies on preparatory set activity mediated by neural pathways linking SMA and striatum that do not employ dopamine. Subsequently failing to replicate this differential effect ${ }^{12}$ they accommodated this failure in yet a further post hoc postulate to the effect that only CRT under conditions of directional uncertainty was dopamine sensitive. While the path taken by Pullman et $\mathrm{al}^{9}$ might hold some potential interest, they seem to have been deflected into it by a number of illfounded statistical inferences. These include acceptance of the null hypothesis concerning medication effects on SRT obtained on only five highly variable patients (it is, incidentally, the large size of their CRT effect rather than the diminutiveness of their SRT effect that appears anomalous in table 1), as well as assuming that because the effect on CRT was significant and that on SRT was not, that difference must, itself, be significant. Such a conclusion could only be sustained by demonstrating a significant interaction between medication and RT task, an analysis that the authors neglected to perform.

\section{TASK MANIPULATIONS}

Reaction time is neither a process nor a neuropsychological test of the integrity of some particular function. It is certainly not a laboratory model of akinesia. ${ }^{3}$ It is merely a composite measure of the speed with which a particular task can be performed. Even the 
most elementary of RT tasks usually requires a complex arrangement of attentional, perceptual, and motor operations that include preparatory activities, stimulus processing, use of working memory for retrieval of stimulus-response mappings and generation of predictions, and decision making. The success with which RT measurement can be brought to bear on specific processes and their integrity depends on experimental manipulations of task variables. If there is a case to be made for the application of RT measures, it must surely lie in the century and a half of experimental exploration of the variables that influence RT, together with attempts at a functional interpretation of the outcomes of these manipulations. ${ }^{1}$ This apparatus has hardly yet been brought to bear on the investigation of information processing defects in Parkinson's disease and their anatomical and pharmacological substrates.

As for the tasks used, the most salient variation is to be found in the nature and complexity of the response required, ranging from an isometric keypress to movements requiring calibrated force and spatial accuracy. Movements that require considerable planning have the potential to show the SRT advantage to great effect but this will only be evident if the subject elects to and is permitted to prepare fully in advance in SRT and is prevented from initiating a response in CRT before programming is complete.

\section{PATIENT SELECTION AND GENERALISATION}

Such is the heterogeneity of patients with Parkinson's disease that a large, unconstrained sample will usually contain enough patients with abnormally prolonged RT to yield a significant effect of group. The question of whether the results of such a comparison attain statistical significance seems to us a relatively uninteresting one, if only because no statement about such a group can be made with certainty that it will hold for all the members. As the disease progresses, cohort heterogeneity probably increases and the effectiveness of levodopa therapy diminishes. Consequently, we would expect a corresponding increase in the likelihood of registering a significant difference between patients with Parkinson's disease and normal age matched controls.

Studies on Parkinson's disease need to be much more explicit about the principles guiding subject selection and generalisability. It is unclear to what extent the inconsistencies in this literature depend on differences in patients or in tasks. In our view, any generalisations which hold strictly and universally over the entire Parkinson's disease population are likely to have substance inversely proportional to their coverage. It is better to be able to assert something specific and testable about a subtype than to construct a probabilistic commentary on the nature or characterisitics of a supposed disease entity.
We are grateful to Sheila Henderson for comments on the manuscript. Thanks are due to Marc Gafen for his assistance with data analysis. Details of this paper have previously been Pharmacological Society. This work was supported by the Wellcome Trust.

1 Posner MI. Chronometric explorations of mind. Hillsdale NJ: Lawrence Erlbaum Associates, 1978.

2 Koster EG, ed. Attention \& performance 2. Amsterdam: North Holland publishing Co, 1968.

3 Jahanshahi M, Brown RG, Marsden CD. Motor slowness in Parkinson's disease. In: Stelmach GE, Homberg V, eds, Sensorimotor impairment in the elderly. The Netherlands: Kluwer Academic Publishers, 1993:265-91.

4 Velasco F, Velasco M. A quantitative evaluation of the effects of L-dopa on Parkinson's disease. Neuropharmacology 1973:89-9.

5 Rafal RD, Posner MI, Walker JA, Friedrich FJ. Cognition and the basal ganglia. Brain 1984;107:1083-94

6 Girotti F, Carella F, Grassi MP, Soliveri P, Marano R, Caraceni $T$. Motor and cognitive performances of parkinsonian patients in the on and off phases of the disease. $f$ Neurol Neurosurg Psychiatry 1986;49:657-60.

7 Bloxham CA, Dick DJ, Moore M. Reaction times and attention in Parkinson's disease. $f$ Neurol Neurosurg attention in Parkinson's dise

8 Viallet F, Masson R, Khalil R. Performance of a bimanual load-lifting task by parkinsonian patients. $f$ Neurol Neurosurg Psychiatry 1987;50:1274-83.

9 Pullman SL, Watts RL, Juncos JL, Chase TN, Sanes JN. Dopaminergic effects on simple and choice reaction time in Parkinson's disease. Neurology 1988;38:249-54.

10 Starkstein S, Leiguardia R. Neuropsychological correlates of brain atrophy in Parkinson's disease: A CT-scan study. Mov Disord 1992;8:51-5.

11 Montgomery E, Nuessen J. The movement speed/accuracy operator in Parkinson's disease. Neurology 1990;40: 269-72.

12 Pullman SL, Watts RL, Juncos JL, Sanes JN. Movement amplitude choice reaction time performance in Parkinson's disease may be independent of dopaminergic status. F Neurol Neurosurg Psychiatry 1990;53:279-83.

13 Jordan N, Sagar H, Cooper J. Cognitive components of reaction time in Parkinson's disease. $\mathcal{f}$ Neurol Neurosurg reaction time in Parkinson'
Psychiatry 1992;55:658-64.

14 Jahanshahi M, Brown R, Marsden CD. The effect of withdrawal of dopaminergic medication on simple and choice reaction time and the use of advance information in Parkinson's disease. $\mathcal{f}$ Neurol Neurosurg Psychiatry 1992 55:1168-76.

15 Brown V, Schwarz U, Bowman E, Fuhr P, Robinson D, Hallett M. Dopamine dependent reaction time deficits in patients with Parkinson's disease are task specific. Neuropsychologia 1993;31:459-69.

16 Lueck CJ, Crawford TJ, Henderson L, Van Gisbergen JAM, Duysens J, Kennard C. Saccadic eye movements in Parkinson's disease: II. Remembered saccades-towards

17 Benecke R, Rothwell JC, Dick JPR, Day BL, Marsden CD Disturbance of sequential movements in patients with Disturbance of sequential movements in patients with
Parkinson's disease. $₹$ Neurol Neurosurg Psychiatry 1987; Parkinson's

18 Welford AT. Single channel-operation in the brain. Acto Psychologia 1967;27:5-19.

9 Wilkinson RT. Response-stimulus interval in choice reaction time. Interaction with sleep deprivation, choice and practice. $Q \mathcal{F}$ Exp Psychol 1990;42A:401-23.

20 Rabbitt PMA. The effects of response stimulus interval duration on serial choice reaction time: Preparation time or response monitoring time? Ergonomics 1980;23:65-77.

21 Harrison J, Goodrich S, Kennard C, Henderson L. The consequences of "frontal" impairment for reaction times in Parkinson's disease. $f$ Neurol Neurosurg Psychiatry in Parkinson's

22 Pascual-Leone A, Valls-Solé J, Brasil-Neto J P, Cohen L G, Hallett M. Akinesia in Parkinson's disease. I. Shortening of simple reaction time with focal, single pulse transcranial simple reaction time with focal, single pulse trans
magnetic stimulation. Neurology 1994;44:884-91

23 Pascual-Leone A, Valls-Solé J, Brasil-Neto J P, Cammarota A, Grafman J, Hallett M. Akinesia in Parkinson's disease. II. Effects of subthreshold repetitive transcranial motor cortex stimulation. Neurology 1994;44:892-98.

24 Zimmerman P, Sprengelmeyer R, Fimm B, Wallesch C-W Cognitive slowing in decision tasks in early and advanced Parkinson's disease. Brain Cogn 1992; 18:60-6.

25 Revonsuo A, Portin R, Koivikko L, Rinne J, Rinne U. Slowing of information processing in Parkinson's disease. Brain Cogn 1993;21:87-110.

26 Goodrich S, Henderson L, Kennard C. On the existence of an attention demanding process peculiar to simple reacCognitive Neuropsychology 1989;6:309-31.

27 Fahn S, Libsch L, Cutler R. Monoamines in the human neostriatum: topographic distribution in normals and in neostriatum: topographic distribution in normals and in
Parkinson's disease and their role in akinesia, rigidity, chorea and tremor. $¥$ Neurol Sci 1971;14:427-55.

28 Mason S, Fibiger H. Noradrenaline and selective attention. Life Sci 1979;25:1949-56.

29 Stern Y, Mayeaux R, Cote L. Reaction time and vigilance in Parkinson's disease. Arch Neurol 1984;41:1086-89. 